\title{
Relevancia de la ejecución experimental de proyectos con microcontroladores en el aprendizaje de la ingeniería electrónica
}

\author{
ERNESTO DÍAZ RONCEROS* \\ Universidad Nacional José Faustino Sánchez Carrión - Perú \\ Recibido el 01-04-19; primera evaluación el 19-12-19; \\ segunda evaluación el 14-02-20; aceptado el 26-02-20
}

\section{RESUMEN}

El objetivo del estudio fue determinar la mejora del aprendizaje de los estudiantes de Ingeniería Electrónica de la Universidad Nacional José Faustino Sánchez Carrión en la asignatura de Microcontroladores, aplicando microrobótica en el octavo ciclo. La población estuvo constituida por todos los estudiantes matriculados en el curso de Microcontroladores, 2016-II. El tipo de investigación fue descriptiva-relacional y el diseño fue transversal-correlacional. Como instrumento para la recolección de datos, se utilizó un pretest y postest, donde se reporta conocimientos sobre la programación de microcontroladores. El 66\% de los estudiantes desaprobó el pretest y el $100 \%$ de los estudiantes aprobó el postest. El aprendizaje de los estudiantes mejoró significativamente con la aplicación de la microrobótica en la asignatura de Microcontroladores, y la actitud de los estudiantes sobre el uso de estos dispositivos en el área de la electrónica y automatización fue positiva.

Palabras clave: microcontroladores, microrobótica, mprendizaje.

\footnotetext{
* Licenciado de Ingeniería Electrónica por la Universidad Nacional José Faustino Sánchez Carrión (UNJFSC), Perú. Magíster en Docencia Superior e Investigación Universitaria por la UNJFSC. Doctorando en Ciencias de la Educación, cursando el tercer ciclo. Docente universitario y asesor de tesis a nivel pregrado. Investigaciones desarrolladas en el área de ingeniería y educación universitaria. Área de especialización: microcontroladores, neumática, electroneumática, y robótica y sistemas expertos. Correo electrónico: ediazronceros@gmail.com
} 
Relevance of the experimental implementation of projects with microcontrollers in the learning of electronic engineering

\section{Abstract}

The objective of the study was to determine the learning improvement of students of Electronic Engineering of the Universidad Nacional José Faustino Sánchez Carrión in the subject of Microcontrollers applying Microrobotics in the eighth cycle. The population consisted of all students enrolled in the microcontroller course, 2016-II. The type of research was descriptive-relational and the design was transverse-correlational. As a tool for data collection, a pres-test and post-test were used where knowledge of microcontroller programming is reported. $66 \%$ of students disapproved of the pre-test and $100 \%$ of students passed the post-test. The student learning improved significantly with the application of the Microrobotics in the subject of Microcontrollers and the attitude of the student on the use of these devices in the area of electronics and automation was positive.

Keywords: Microcontrollers, Microrobotics, learning.

Relevância da execuçáo experimental de projetos com microcontroladores no aprendizado da engenharia eletrônica

\section{Resumo}

O objetivo do estudo foi determinar a melhoria do aprendizado dos estudantes de Engenharia Eletrônica da Universidade Nacional José Faustino Sánchez Carrión na disciplina de Microcontroladores aplicando microrobotics no oitavo ciclo. A população foi constituída por todos os estudantes matriculados no curso de Microcontroladores, 2016-II. O tipo de investigação era descritivo-relacional e o desenho foi transversal-correlacional. Como instrumento para coletar os dados foi usado o pre-teste e o post-test onde foram informados conhecimentos sobre a programação de Microcontroladores. O $66 \%$ dos estudantes desaprovaram o pre-teste e $100 \%$ dos estudantes aprovaram o post-teste. A aprendizagem do estudante melhoro siginificativamente com a aplicação da microrobótica na disciplina de Microcontroladores e a atitude do estudante sobre o uso destes dispositivos na área da eletrônica e da automatização foi positiva.

Palavras-chave: microcontroladores, microrobótica, aprendizagem. 


\section{INTRODUCCIÓN}

Los microcontroladores, hoy en día, cumplen un rol muy importante en la sociedad. Estos pequeńos procesadores son utilizados en diversos sistemas de tecnología como: un smartphone, una laptop, un smart tv, una lavadora, hasta procesos de automatización industrial, ya sean para el control de humedad, temperatura, presión, distancia, etc.

Romero, Angulo y Angulo (2006) mencionan que los conocimientos sobre microcontroladores contribuyen en las áreas del campo laboral para los perfiles académicos de los ingenieros en las especialidades de informática, electrónica, automática o de telecomunicaciones. Esto se debe al auge y su utilización masiva en la industria. El conocimiento de programación de estos chips y sus periféricos integrados pueden abrirle las puertas del mundo laboral a muchos de nuestros estudiantes. Asimismo, Bono y Martín (2003) afirman que «los microcontroladores son uno de los componentes cruciales que más están experimentando un gran aumento de potencia debido al imparable desarrollo tecnológico» (p. 1).

Entonces es muy importante para alguien que estudia o se especializa en esta área de la automatización conocer sobre los microcontroladores y aprender a programarlos, es por ello que en la carrera profesional de Ingeniería Electrónica de la Universidad Nacional José Faustino Sánchez Carrión se dicta la asignatura de Microcontroladores.

En el dictado de la asignatura de Microcontroladores en los ciclos 2015-I y 2015-II, se ha observado que los estudiantes perciben como complejo el tema de programación relacionado a los microcontroladores. El factor que condiciona esta idea en los estudiantes es no tener una experiencia previa, por lo tanto, asumen que el curso será muy teórico y tedioso. Si bien para empezar a programar se debe conocer los fundamentos básicos (teóricos), esto no debe reflejarse en todo el ciclo y siempre es necesario llevarlo a la práctica. Por ello, para lograrlo, se pueden implementar o realizar diversos proyectos de los cuales los más interesantes y que generan mayor motivación son los relacionados a la microrobótica.

Con los hallazgos de la presente investigación se creará y demostrará que la microrobótica se puede aplicar como una estrategia de aprendizaje en la asignatura de Microcontroladores, facilitando así la enseńanza del estudiante, quien a través de la microrobótica comprenderá mejor los conceptos planteados en clase.

La justificación central de esta investigación se basa principalmente en la utilidad de la metodológica activa de aprendizaje que tiene el desarrollar 
un prototipo (Microrobot) como proyecto final para la asignatura de Microcontroladores, en la cual el estudiante observará de forma práctica el funcionamiento de estos y el grado de autonomía que se le pueda dar al microrobot con la programación realizada, como justificación secundaria que genere motivación e incentive el interés del estudiante en el ámbito de la investigación, ya que realizar un proyecto de estas características necesita que el estudiante adquiera información adicional para aplicarlo.

Por lo tanto, nuestra hipótesis será que los microcontroladores se relacionan significativamente de forma positiva con la microrobótica en los estudiantes de octavo ciclo de ingeniería electrónica de la Universidad Nacional José Faustino Sánchez Carrión.

El objetivo de la investigación es determinar la mejora del aprendizaje de los estudiantes de ingeniería electrónica de la Universidad Nacional José Faustino Sánchez Carrión en la asignatura de Microcontroladores aplicando microrobótica en el octavo ciclo.

\section{MarCo teÓRICo}

\subsection{Antecedentes del estudio}

Los estudios que se presentan son del tipo antecedentes de campo, es decir, las investigaciones son de tipo experimental y se realizaron con sujetos donde se recaudan datos numéricos o información descriptiva. Asimismo, están relacionados a los microcontroladores y su aprendizaje, basado en proyectos que en algunos casos se utiliza la robótica como base para formular el proyecto, y, en otros, se puede optar por las diversas ramas de la electrónica, como son telecomunicaciones, instrumentación, electrónica digital, procesamiento digital de señales, etc.

Najid, Ridzwan y Tee (2018) realizaron una investigación teniendo por finalidad observar la implementación del aprendizaje basado en problemas $(\mathrm{ABP})$ en el campo de la ingeniería para promover el aprendizaje permanente en la transmisión de conocimientos. Indican que normalmente el método de aprendizaje practicado para la materia de Microcontrolador se realiza mediante los manuales y libros de texto correspondientes, haciendo que los estudiantes memoricen los términos técnicos difíciles, generando que descuidan el propósito real de los términos usados en la materia de Microcontrolador. Analizaron la literatura de otros autores que emplean el $\mathrm{ABP}$ de los cuales resumen que es un método eficaz de aprendizaje en ingeniería y campos técnicos; asimismo, enfatizan características de aprendizaje basadas en situaciones del mundo real. 
De esta manera, los estudiantes tienen la oportunidad de formarse para aprender técnicas de autoaprendizaje que son elementos importantes para su futura carrera. Adicionalmente, pueden mejorar sus resultados de aprendizaje mediante el trabajo en equipo y fomentar la confianza mediante actividades realizadas en el aula. Se deduce que el método ABP puede producir alumnos más motivados, construir una comprensión más profunda del mundo real, fomentar el autoaprendizaje, mejorar el pensamiento crítico, desarrollar habilidades cognitivas, mejorar las habilidades de resolución de problemas, trabajo en equipo y habilidades de comunicación. En conclusión, afirman que el ABP es un método de aprendizaje que puede nutrir el interés de los estudiantes en el aprendizaje de la ingeniería de una manera más interesante y eficaz.

Cruz (2017) realizó una investigación con el objetivo de desarrollar competencias profesionales en los estudiantes de ingeniería mecánica aplicando como estrategia docente el ABP. Metodológicamente, se basó en aspectos cualitativos observando a los estudiantes durante el desarrollo del proyecto, por otra parte, la población estuvo constituida por 36 estudiantes de la asignatura de Electrónica Digital Aplicada a quienes se les aplicaron los instrumentos de recolección de datos que fueron la observación y rúbricas de evaluación, también se indica que la investigación se realizó en cinco fases: análisis de la asignatura, diseño del proyecto, implementación del proyecto, análisis de datos y redacción del informe final. Como resultados se muestra que un $77,78 \%$ de la población estudiantes logró desarrollar elementos cognitivos y el 22,22\% tiene un nivel autónomo mínimo para la investigación; con respecto al nivel de dominio estratégico, el 77,78\% alcanzó este nivel mediante las actividades planteadas en el ABP. Finalmente, el investigador concluye afirmando que el diseńo y aplicación de la metodología de ABP favorece en los estudiantes el desarrollo de competencias profesionales establecidas en el programa de Electrónica Digital Aplicada; asimismo, el estudiante, mediante la implementación del proyecto, construyó su conocimiento al relacionar la aplicación con los temas de la asignatura.

Sababha, Alqudah, Abualbasal y AlQaralleh (2016) efectuaron un estudio cuyo objetivo fue aplicar el aprendizaje basado en proyectos para mejorar la enseñanza del curso de Sistemas Integrados en PSUT (Princess Sumaya University for Technology). La población estuvo constituida por los 41 estudiantes de la clase en el semestre de otońo de 2013. Como instrumento, se utilizó una encuesta donde respondieron a seis preguntas principales, las cuales tenían como fin medir los beneficios obtenidos por los estudiantes a través de la experiencia de los proyectos. Se pidió que dieran a cada pregunta una clasificación en una escala de 0 a 5 , donde 5 significaba «totalmente de acuerdo». 
De los resultados de la encuesta realizada a los estudiantes, un 97,5\% afirma que el proyecto ha reforzado los conceptos aprendidos en la clase, 90,2\% estaban de acuerdo en que el aprendizaje basado en proyectos aumentaba su interés por la ingeniería eléctrica, $92,7 \%$ indica que el interés por los sistemas integrados se elevó mediante el aprendizaje basado en proyectos, 93,7\% concuerda que tener un problema de la vida real en el proyecto lo hizo más interesante, $80,5 \%$ de los estudiantes estaban de acuerdo en que trabajar en el proyecto con su grupo aumentaba sus capacidades de trabajo en equipo, y el 94,1\% indicó que el proyecto merecía su tiempo y esfuerzo. Finalmente, concluyeron que este método de ABP en la enseñanza del curso ha contribuido a salvar la brecha entre la enseñanza teórica de ingeniería eléctrica y el mundo real. Los comentarios de los estudiantes confirmaron la eficacia del método presentado para mejorar la comprensión y la capacidad de ellos en la aplicación de conceptos de diseño de sistemas integrados para resolver problemas de ingeniería en el mundo real.

Díaz, Jeraldo y Tapia (2013) desarrollaron un estudio descriptivo con el objetivo de integrar emisores de luz, de sonidos y sensores, basados en un programa para microcontroladores que permita registrar datos o emitir información para dar cuenta de fenómenos y diseñar estrategias didácticas basadas en el enfoque ciencia, tecnología y sociedad (CTS) y en la enseńanza de las ciencias basada en la indagación (ECBI) para el estudio de la física en Educación Media. La muestra está conformada por los estudiantes del seminario y el instrumento utilizado fue las escalas de apreciación. De los resultados obtenidos, a nivel general, se señalan que todas las guías fueron aprobadas por los docentes que las evaluaron. Finalmente, concluyen que, partiendo de la opinión entregada por los docentes, se puede reafirmar algunos criterios adoptados en la elaboración de los manuales y guías orientadas al profesor, reconociendo que el uso de estos componentes como emisores y sensores es una propuesta integradora e innovadoras de las habilidades de pensamiento, y acercando situaciones científicas cotidianas al lenguaje de las y los estudiantes. Sin embargo, hay que resaltar que algunas guías deberán ser modificadas para lograr una mejora de los porcentajes de aprobación en el material elaborado. Con respecto a las preguntas que se plantearon al inicio de este seminario, señalan que la forma en que se puede incorporar el uso de microcontroladores y sensores en la enseñanza de la física es mediante la experimentación con el uso de la secuencia indagatoria ECBI.

Cifuentes (2011) realizó una investigación descriptiva donde busca identificar las didácticas y enfoques pedagógicos que emplean los docentes de las universidades Militar Nueva Granada y Nacional de Colombia para la enseńanza de los microcontroladores, para ello, estudió 22 estudiantes 
pertenecientes a la Universidad Nacional y a 35 de la Universidad Militar para un total de 57, para realizar el estudio se utilizó como instrumento la encuesta. El estudio reveló que tanto los proyectos como su aplicación para la solución de problemas son de gran importancia y concluyó afirmando que los proyectos que solucionan problemas en la vida real son un excelente estímulo tanto para alumnos como profesores al momento de generar aprendizaje significativo y todo esto relacionado con los microcontroladores.

Arias, Rodríguez, Lamar, Linera y Hernando (2010) realizaron una investigación descriptiva y plantearon las prácticas de una asignatura de diseño digital, basado en un microcontrolador desde la perspectiva del aprendizaje basado en problemas. La muestra que utilizaron fue de 100 estudiantes pertenecientes a la Universidad de Oviedo en España, y como instrumento usaron el test y evaluaciones, de los cuales al obtener los resultados afirman que es posible que exista una relación entre el grado de dedicación a las prácticas y la nota general obtenida en el curso. Asimismo, la dinámica del ABP presenta resultados buenos no solo cuando el proyecto que se plantea a los alumnos es de gran complejidad y único, sino también cuando se plantean numerosos proyectos de menor relevancia centrados en temas específicos de la asignatura; sin embargo, es de vital importancia en este caso reducir el tamaño de los integrantes de trabajo para evitar que estudiantes pasivos aparezcan.

Oliver, Toledo, Pujol, Sorribes y Valderrama (2009) hicieron un estudio descriptivo con el objetivo de presentar un mecanismo vertebrado basado en $\mathrm{ABP}$ en robótica aplicado a las materias de hardware y software para los estudiantes en los primeros cursos de la ingeniería informática. La muestra estuvo conformada por los alumnos de l'Escola Tècnica Superior d'Enginyeria y l'Escola Tècnica en Informàtica de Sabadell. El instrumento utilizado fue Programa de ABP y, de los resultados, se lograron implementar el ABP con conocimientos y competencias previstas del ABP con robótica en primer curso de informática. Por último, señalan que las ventajas que ofrece el desarrollo de un $\mathrm{ABP}$ adecuado a los estudiantes de los primeros cursos serán el trabajo para desarrollar un proyecto común que les permita interactuar en cada etapa, unificar los conocimientos de hardware y software, introducir las etapas de programación iniciando desde un lenguaje ensamblador hasta llegar a desarrollar aplicaciones complejas en lenguajes de alto nivel, comprender en qué consiste la programación en tiempo real, y así el estudiante entiende los aspectos de ingeniería (2009, p. 5).

Macías-Guarasa, Montero, San-Segundo, Araujo y Nieto-Taladriz (2006) efectuaron un estudio con un enfoque para diseñar currículos de sistemas electrónicos y, de esta manera, hacer que la electrónica sea más atractiva para los 
estudiantes. El enfoque que proponen se basa en el desarrollo de proyectos multidisciplinarios utilizando el aprendizaje basado en proyectos y estrategia para aumentar el atractivo del currículo. La estructura curricular consta de ocho cursos: cuatro cursos teóricos y cuatro cursos de ABP (incluyendo una tesis de Maestría obligatoria). En los cursos de aprendizaje basado en proyectos los estudiantes, trabajando juntos en grupos, desarrollaron sistemas multidisciplinarios, que se vuelven cada vez más complejos. Afirman que el currículo fue evaluado con éxito durante los últimos cuatro años académicos; asimismo, los estudiantes han aumentado su interés por la electrónica y han dado a los cursos un grado medio de más del $71 \%$ para todas las evaluaciones de cursos de ABP, los cuales fueron obtenidos con el instrumento de la encuesta. A su vez, adquirieron nuevas habilidades y muy buenos resultados académicos: la nota promedio fue de más del $74 \%$ para todos los cursos de ABP. Un resultado importante que se debe resaltar de esta investigación es que todos los estudiantes han desarrollado sistemas electrónicos más complejos y sofisticados. Por último, señalan que el enfoque para diseñar currículos de sistemas electrónicos, empleando el aprendizaje basado en proyectos, hacen que la electrónica sea más atractiva para los estudiantes.

\subsection{Bases teóricas}

\subsubsection{Microrrobótica}

Es parte de la ingeniería robótica, la cual comprende desde la micromanipulación de objetos que se encuentran en rangos de milímetros a micras hasta el diseño y elaboración de microrrobots autónomos. Actualmente, existen diversas maneras de describir y definir a un microrrobot, así como sus características. Asumiendo que se trata de un sistema de reducidas dimensiones, es importante conocer el concepto de este para entender lo que es un microrrobot (Olivares, 2011).

Romero, Angulo y Angulo (2006) indican que la microrrobótica es una tecnología actual que se centra en la aplicación de pequeńos robots móviles o microbots que previamente son programados y dotados de los correspondientes sensores y actuadores para resolver, de modo optimizado, diversas tareas, tales como vigilar, limpiar, explorar, etc.

Un microrrobot es un robot móvil programable de dimensiones pequeñas que realiza tareas sencillas, está conformado por un microcontrolador que se encarga de gobernar todos sus movimientos y, según el trabajo encomendado, ejecuta un programa. Asimismo, consta de entradas y salidas de información para los actuadores (Microsystems Engineering, 2005). 
Figura 1. Microrrobot Home Boe-bot. Ingeniería de Microsistemas Programados S.L.

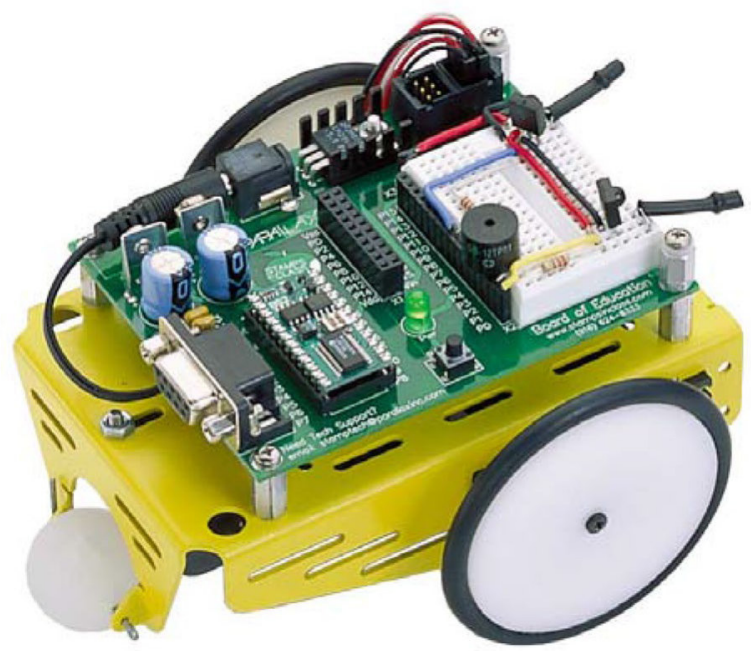

Fuente: Microsystems Engineering (2005).

1. Está constituido de los siguientes elementos:

2. Estructura mecánica que actúa de soporte para los componentes.

3. Motores acondicionados junto con ruedas

4. Sensores que recogen información del entorno de variables como temperatura, luz, presencia de obstáculo, etc.

5. Actuadores como altavoz, motor, LED, display, zumbador, etc.

6. Tarjeta de control que contiene al microcontrolador y su programa de instrucciones, el cual se encarga de darle funcionabilidad a todo el sistema del microrrobot.

\subsubsection{El microcontrolador}

Reyes (2008) afirma que el microcontrolador es un circuito integrado que posee un su interior una arquitectura similar a la de un computador, como memorias RAM, EEPROM, CPU y periféricos de entrada y salida o también llamados I/O. 
Un microcontrolador recién salido de fábrica no ejecutará ninguna tarea. Este deberá ser programado para realizar desde un pequeńo encendido de un foco led hasta un sistema avanzado de control de un robot. Tiene la capacidad de reemplazar una gran cantidad de circuitos lógicos como compuertas AND, OR, NOT, NAND, temporizadores, decodificadores, conversores A/D - D/A, etc., simplificando el diseño a una placa de circuito impreso (PCB, por sus siglas en inglés) de reducida dimensión y pocos componentes. Arquitectura Interna: Los microcontroladores poseen la mayoría de componentes de un computador, no obstante, se diferencian en que estos cuentan con características fijas que no se alteran Reyes (2008).

Figura 2. PIC 16F876A

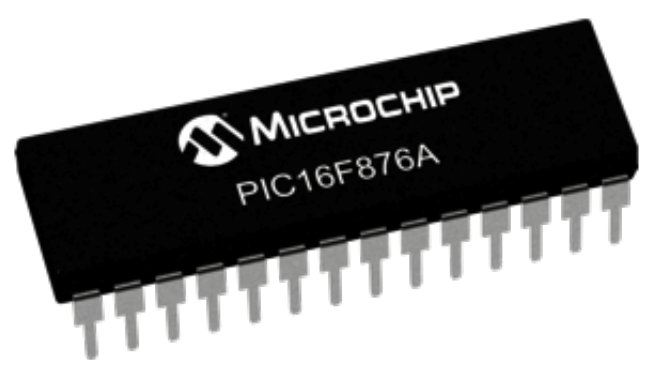

Fuente: Microchip, s.f.

\subsubsection{Partes principales de un microcontrolador}

Las partes principales de la arquitectura interna de un microcontrolador son las siguientes:

a) Procesador. Debido a que es necesario obtener excelentes rendimientos en el procesamiento de las instrucciones, esto ha conllevado a la migración de procesadores de arquitectura tradicional tipo Von Neumann (ver Figura 3) a una arquitectura Harvard (ver Figura 4); esta última se caracteriza y diferencia, porque la CPU se conectaba con una única memoria donde se almacenan tanto datos como instrucciones mediante un sistema de buses (Angulo y Angulo, 2003). 
Figura 3. Arquitectura de Von Neuman

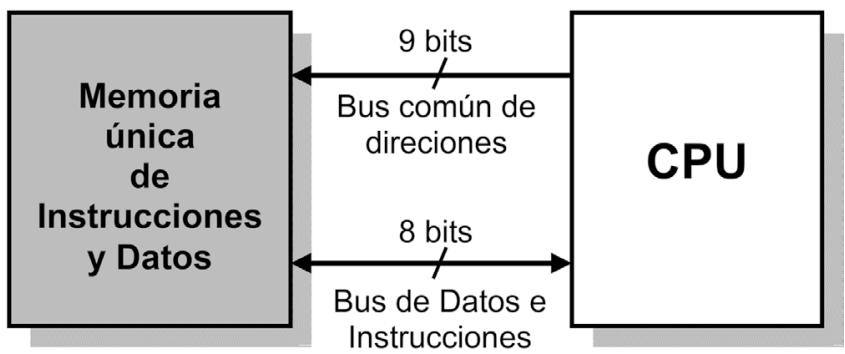

Fuente: Reyes, 2008.

La memoria Harvard consiste en tener dos memorias de forma independiente: la de datos y la de instrucciones. Cada una de ellas cuenta con su propio sistema de buses de acceso, en consecuencia, esta característica dual nos permite generar el paralelismo y proporciona el acondicionamiento del tamaño de las palabras y los buses a los requerimientos específicos de las instrucciones y de los datos; sin embargo, la cantidad de cada memoria es difiere entre ellas (Romero, Angulo y Angulo, 2006).

Figura 4. Arquitectura Harvard

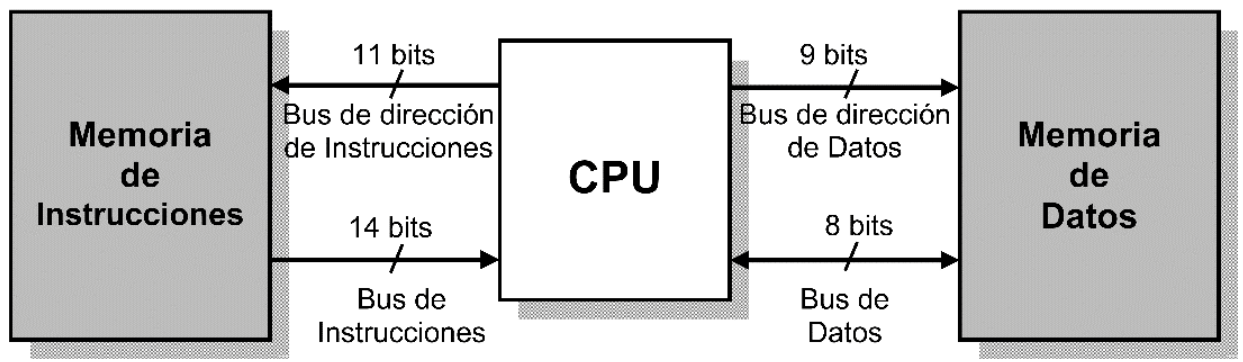

Fuente: Reyes, 2008.

Romero, Angulo y Angulo (2006) afirman que, en la actualidad, la mayoría de procesadores están conformados por una arquitectura tipo RISC (computadoras de juego de instrucciones reducido). Esta arquitectura se caracteriza por tener un repertorio de instrucciones máquina reducida y pequeña, de tal manera que el mayor conjunto de instrucciones que se logrará ejecutar es un ciclo de instrucción. 
b) Memoria no volátil para contener el programa. Los microcontroladores están diseñados con el fin de que almacenen, en su memoria de programación, las instrucciones del programa de control, no se permite usar memorias externas para ampliar y como se ejecutará siempre el mismo programa debe estar grabado de manera permanente (Angulo y Angulo, 2003).

c) Memoria de lectura y escritura para guardar datos. Información que procesan los programas varía continuamente, y por lo que se requiere que la memoria que los contiene debe ser de lectura y escritura, la memoria RAM estática (SRAM) resulta ser la adecuada, aunque sea volátil. Existen microcontroladores que tienen como memoria tipo EEPROM para la lectura y escritura de datos, no volátil, el fin de usar esta memoria es si sucede un corte en el suministro de la energía no ocasionará perdida de información, ya que estará disponible al reiniciar el microcontrolador (Angulo y Angulo, 2003).

d) Lineas de entrada y salida para los controladores periféricos. A diferencia de los pines de conexión que reciben alimentación, se tiene otras dos para el cristal de oscilación que regula la frecuencia de trabajo y un pin más para configurar el reset, los demás pines del microcontrolador se utilizan para la comunicación externa con los periféricos (Angulo y Angulo, 2003). Las líneas se adaptan mediante E/S con los periféricos, estos conducen la información en paralelo y se agrupan en conjuntos de bytes recibiendo el nombre de Puertos, hay series con periféricos que soportan la comunicación en serie; otros tienen conjuntos de líneas que implementan puertos de comunicación para otros protocolos como el I2 ${ }^{\circ} \mathrm{C}$, el USB, etc. (Angulo y Angulo, 2003).

e) Recursos auxiliares. Según las aplicaciones a las que orienta el fabricante, cada modelo de microcontrolador incorpora una diversidad de complementos que refuerzan la potencia y la flexibilidad del dispositivo (Angulo y Angulo, 2003).

\subsubsection{Programación de Microcontroladores}

Los lenguajes de bajo nivel, o también llamados máquinas, representan un gran ahorro de código en el desarrollo de los programas debido a que este lenguaje trabaja directamente con los registros, lo que resulta de gran importancia por la estricta limitación de memoria de instrucciones, un programa en lenguaje ensamblador bien realizado será ejecutado con gran eficiencia (Angulo y Angulo, 2003). 
Los lenguajes de programación como el C y el BASIC considerados de alto nivel son los más empleados para programar microcontroladores, asimismo, existen diversas empresas que desarrollan compiladores e intérpretes para la diversa gama de microcontroladores (Angulo y Angulo, 2003).

Figura 5. Lenguajes de programación

LENGUAJE HUMANO

Lenguaje de alto nivel
Lenguaje de bajo nivel

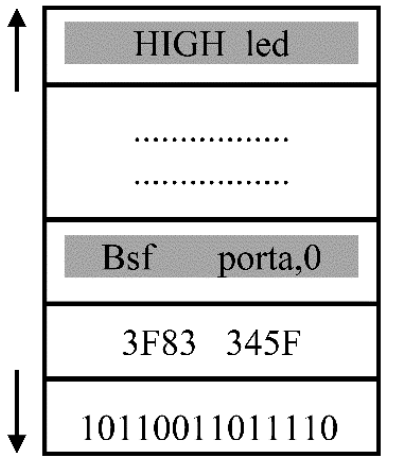

Lenguaje BASIC

Otros lenguajes

LENGUAJE DE MAQUINA

Fuente: Reyes, 2008.

\subsubsection{Consideraciones básicas para montar un proyecto con PIC}

Reyes (2008) considera que para montar un proyecto con PIC es importante conocer las consideraciones básicas y su estructura del microcontrolador para no poner en riesgo al mismo.

- Los microcontroladores tienen tecnología CMOS. Esto nos indica que consume muy poca corriente, pero que a la vez resulta ser susceptible a daños por estática. Se recomienda, por lo tanto, utilizar pinzas para manipularlos y así poder transportar el PIC desde el grabador al protoboard o viceversa, o en su defecto utilizar un guante antiestático (Reyes, 2008).

- Utilice un regulador de voltaje como el CI 7805 que nos entrega exactamente $5 \mathrm{~V}$ y no un adaptador de pared o cargador de celular, ya que la salida de voltaje no siempre será la misma que indica su fabricante. Finalmente, puede utilizar un circuito con un diodo zener de 5.1 V (Reyesm, 2008). 
- No sobrepasar los niveles de la corriente, tanto en la entrada como en la salida, recordar que el microcontrolador puede proporcionar por cada pin una corriente máxima de $25 \mathrm{~mA}$. de igual manera, soporta en la entrada una corriente máxima de $25 \mathrm{~mA}$, en otras palabras, puede prender un led con una resistencia de $330 \Omega$ (Reyes, 2008).

Reyes (2008) afirma que el voltaje de salida de un pin del PIC si es alimentado con 5 voltios y deberá tener una tensión de 5 voltios. La corriente que necesita un led para un normal encendido es $15 \mathrm{~mA}$. ¿Qué resistencia será necesaria para prender el led correctamente?

$$
V=R \times I
$$

Reemplazando en la ecuación (1) los valores de la tensión e intensidad.

$$
5 \mathrm{~V}=R \times 15 \mathrm{~mA}
$$

Se obtiene el valor de la resistencia: $R=333,33 \Omega \approx 330 \Omega$

En algunos proyectos, será necesario implementar en el circuito un condensador de $0,1 \mu \mathrm{F}$ o $1 \mu \mathrm{F}$ en paralelo al PIC, este evitará un mal funcionamiento que podría ocurrirle, especialmente cuando se utiliza keypads y se conecta adicionalmente un buzzer activo (parlante activo o chicharra) y relés (Reyes, 2008).

Cuando es necesario precisión en el desenvolvimiento del PIC (comunicación serial, tonos DTMF, etc.), se recomienda usar un cristal externo de 4 $\mathrm{MHz}$ o mayor, ya que el oscilador interno RC que tiene el PIC no presenta buena precisión. En un experimento realizado, se conectó dos PIC idénticos usando el mismo programa el cual consistió en un parpadeo de un led con intervalos de un segundo, ambos PIC utilizaron la misma fuente y al momento de iniciar los dos parpadeos eran iguales, al transcurrir unos minutos los leds tenían un comportamiento desigual. Esto demostró que la calibración interna no es el mismo en todos los microcontroladores, si se utiliza cristales externos de $4 \mathrm{MHZ}$ en ambos PIC, no se presentarían comportamiento desigual, esto debido a la precisión de los cristales en cuanto a la frecuencia que entregan (Reyes, 2008).

Finalmente, Reyes (2008) menciona que con las pautas brindadas y tomando como guía el diagrama se puede realizar el encendido de un led (ver Figura 6) sin que el microcontrolador sufra algún daño. 
Figura 6. Diagrama para conectar un PIC con un LED y un pulsador
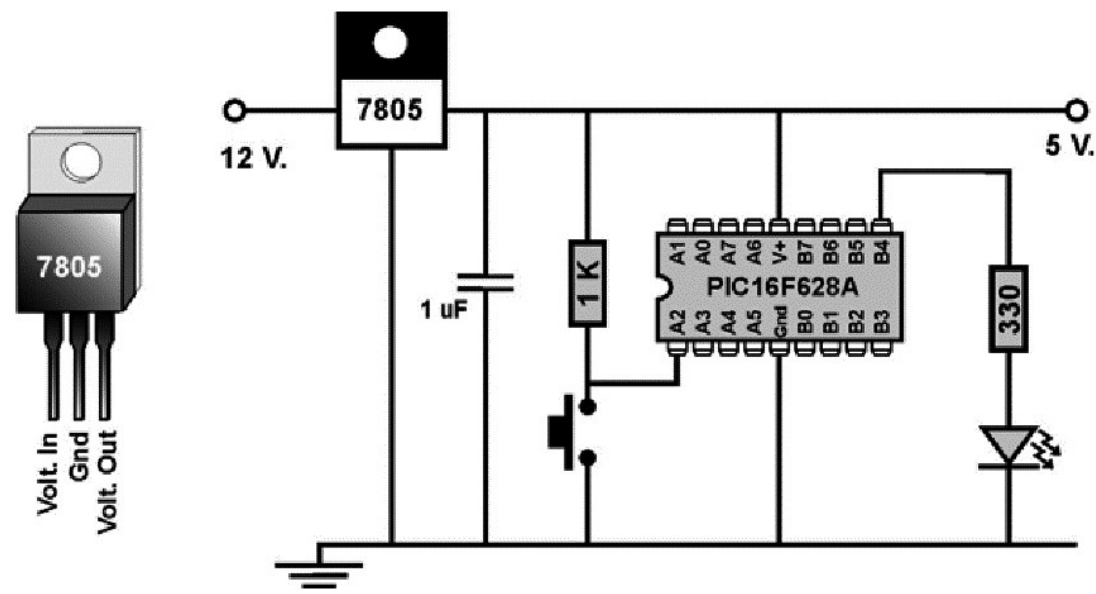

Fuente: Reyes, 2008,

\subsubsection{Robot seguidor de línea}

Un robot seguidor de línea viene a ser robot con autonomía capaz de realizar un recorrido sobre una línea blanca o negra y estar sobre la misma sin perderse en el camino, solo haciendo uso de sensores. Este tipo de robot se utiliza en la industria en las áreas de almacenes y transporte logrando excelentes resultados y realizando modificaciones según el caso, como viene a ser el uso de sensores inductivos para detectar barras metálicas en el suelo (Contreras, 2014).

\subsubsection{Sistema de comunicación}

Bolton (2006) afirma que actualmente los sistemas de comunicación obligan al docente y al alumno de ingeniería a involucrarse con estas tecnologías, existen variadas tecnologías de comunicación como, Infrarrojo, Serial, Bluetooth, Radio Frecuencia, I2C, Ethernet.

\subsubsection{Aprendizaje basado en problemas}

Barrows (1986, como se citó en Morales y Landa, 2004) define al ABP como un método cuyo aprendizaje se basa en el uso de problemas como inicio para el desarrollo de conocimientos, adquiriéndolos e integrándolos a los previos. 


\subsubsection{Aprendizaje basado en proyectos}

El aprendizaje basado en proyectos consta de formar equipos con diversos perfiles, habilidades, áreas disciplinares, idiomas y culturas que en conjunto realizaran proyectos con el fin de solucionar problemas reales. Este tipo de aprendizaje brinda muchas oportunidades para generar conocimiento y permite al estudiante crecer en un entorno donde debe confraternizar con sus compañeros, desarrollando habilidades blandas. Para que los resultados sean productivos, bajo el aprendizaje basado en proyectos, se requiere un diseño instruccional definido, definición de roles y fundamentos de diseño de proyectos (Galeana, 2006).

\subsubsection{Diseño de proyectos}

En la etapa del análisis y la planeación de los proyectos, se debe formular y definir el objetivo, limitar el problema o situación a resolver, identificando los perfiles de los actores que se encuentran involucrados, etc. (Galeana, 2006).

\subsection{Definición de términos}

- Ballcaster. Es una esfera que se sitúa en el punto de equilibrio del robot, pues las dos llantas no son suficientes para darle equilibrio, formando así un triángulo. Su utilidad también radica en reducir el rozamiento con la superficie. Mediante esta esfera, al ser lisa, permite que el microrrobot se desplace de una mejor manera en la pista de competencia.

- Borneras. Los bornes son los contactos que se utilizan para trasmitir la energía que es proporcionada por una fuente de alimentación, se identifica la polaridad con los colores rojo y negro o con los signos positivo y negativo que traen grabados en cada borne o conexión de las fuentes de alimentación.

- Driver L293D. Es un controlador de giro para motores DC, mediante este driver el microcontrolador puede enviarle señales digitales y se puede realizar combinaciones de giros en el robot tales como derecha, izquierda, adelante, retroceso y detenerse.

- OPAMP LM393. Amplificador operacional usado para comparar dos señales analógicas distintas y obtener un estado lógico. En nuestro proyecto, conjuntamente con los potenciómetros, nos permiten calibrar a los sensores para obtener un resultado correcto de acuerdo a las condiciones ambientales que se presenten en el momento. 
- MAX232. Circuito integrado que se emplea en la comunicación serial RS232. Este componente permite acondicionar conjuntamente con capacitores un sistema de comunicación con los microcontroladores directamente en la placa sin necesidad de usar uno externo de un grabador, de tal manera que nos permita grabar la programación de forma directa mediante un cable RS232 a USB conectado al ordenador.

- Motorreductor. Pequeños motores (monofásicos o trifásicos), reductores de engranajes y a menudo variadores de velocidad. Estos motorreductores también se caracterizan por el alto torque que poseen debido a los engranajes que se han acoplado al motor, a nuestro microrrobot le va a dar la fuerza necesaria para mover toda la estructura del chasis, las placas electrónicas e incluyendo las baterías.

- PWM. Genera señales de modulación de ancho de pulso, asimismo, es un módulo que se encuentra en el microcontrolador elegido para el proyecto, permite al robot aumentar y disminuir la velocidad de sus motores, según lo requiera en la pista de competencia. Como ejemplo, si se encuentra en una línea recta, se podrá acelerar al microrrobot y, si se encuentra en una curva, se tendrá las opciones de disminuir su velocidad y evitar derrapes.

- Reprogramable. Característica que tienen los microcontroladores para poder modificar sus programas sin alteración física en sistema electrónico. El robot cuenta con la característica que puede adaptarse en competición a diversas pistas para la categoría seguidores de línea, ya que el Microcontrolador puede ser reprogramado de acuerdo a las condiciones de la pista y ambientales en la cuales deba desarrollarse la competencia.

- Robot. Mecanismo programable en dos o más ejes con un grado de autonomía moviéndose dentro de su entorno para realizar tareas previstos. También es considerado una máquina programable capaz de ejecutar diversas operaciones de manera automática, sustituyendo al personal en algunas tareas, especialmente las que son pesadas, peligrosas o repetitivas; estos pueden estar dotados de sensores, que le facilitan y permiten adaptarse a nuevos entornos.

- Robot seguidor de línea. Un robot seguidor de línea cumple la función de realizar un recorrido de forma autónoma, previamente programado, en una pista con líneas negras y espacios blancos; con el objetivo 
de terminarlo en el menor tiempo posible o lograr el mayor avance posible; haciendo uso solamente de sensores que detecten estos dos colores y utilizando un microcontrolador para tomar acciones sobre el driver de los motores y asignarle una dirección.

- Robótica. Es la ciencia y práctica del diseño, fabricación y aplicación de los robots, asimismo, se define como la técnica que se aplica en el diseño y la construcción de robots y aparatos que realizan trabajos u operaciones, generalmente en plantas industriales y en sustitución del personal de mano de obra.

- Robot móvil. Un robot móvil puede ser una plataforma móvil con o sin manipuladores, son capaces de movilizarse en su entorno y no se quedan fijos en una sola ubicación; son muy importantes en la investigación que se realizó y la mayoría de las universidades tiene uno o más laboratorios, en los cuales se desarrollan investigaciones relacionadas a los robots móviles. Se pueden encontrar también en la industria y los servicios en general como asistentes en hogares complementado a la domótica.

- Sensor CNY70. Sensor infrarrojo de corto alcance, basado en un emisor de luz y un receptor. Estos sensores nos permiten testear el recorrido del microrrobot, siendo así un elemento muy importante, ya que si ellos no se podrían detectar los caminos que debe seguir para completar el recorrido. Existen de diversas calidades y la diferencia radica en la sensibilidad que pueden presentar.

\section{Metodología}

La investigación corresponde a un estudio de tipo descriptivo relacional porque nos permite determinar la relación que existe entre los microcontroladores y la microrrobótica. La población estuvo constituida por los estudiantes del VIII ciclo de ingeniería electrónica, matriculados en la asignatura de Microcontroladores, semestre académico 2016-II, Universidad Nacional José Faustino Sánchez Carrión, Huacho, provincia de Huaura, departamento de Lima. Se utilizó como instrumento de recolección de datos un pretest y postest para determinar el grado de conocimientos que tenían los estudiantes antes y después de aplicar la microrrobótica. La construcción del instrumento requirió identificar indicadores útiles y sencillos que reflejen los aprendizajes de los estudiantes, estos indicadores de logro son señales, rastros observables 
del rendimiento que dan cómputo externamente de lo que está sucediendo en el estudiante y que exige una interpretación y comprensión pedagógica de parte del instructor. De forma adicional, también se elaboró sesiones de clase con sus respectivos diseńos de actividades de aprendizaje, para la enseñanza de la programación e implementación de los microrrobots.

Las sesiones de clase se diseñaron sobre la base del currículo por competencias, por lo tanto, se incluyen las actitudes y capacidades para el estudiante.

- Nombre de la sesión. Ejemplo: SESIÓN VI: PROGRAMACIÓN PARTE I

- Datos informativos. Ubicación donde se desarrolla la sesión, el nombre de la asignatura y la cantidad de horas.

- Competencia. Indicar que es lo que se pretende aprender en la sesión de clase. Ejemplo: Comprender las configuraciones básicas de la programación para el microcontrolador del robot seguidor de linea, desarrollados en la plataforma Proton IDE.

- Capacidad. De los conocimientos adquiridos en las competencias debe cumplir ejercicios propuestos por del docente, también llamado nivel de logro. Ejemplo: A partir de los conocimientos sobre programación, realiza las declaración y simbolización de las variables, puertos del microcontrolador y cristal de oscilación.

- Actitudes. Se analiza el grado de importancia que le da el estudiante a la sesión de clase y al trabajo en equipo, el docente realiza este procedimiento mediante comportamientos observables.

- Contenidos. Se revisa la bibliografía de los temas tratados en la sesión de clase.

\section{Resultados}

Tabla 1. Notas del pretest y postest

\begin{tabular}{ccc}
\hline $\mathrm{N}^{\circ}$ & Pretest & Postest \\
\hline 1 & 08 & 12 \\
2 & 10 & 16 \\
3 & 12 & 16 \\
4 & 18 & 20 \\
5 & 14 & 18
\end{tabular}


Relevancia de la ejecución experimental de proyectos con microcontroladores

\begin{tabular}{ccc}
\hline $\mathbf{N}^{\circ}$ & Pretest & Postest \\
\hline 6 & 08 & 16 \\
7 & 08 & 16 \\
8 & 10 & 14 \\
9 & 18 & 20 \\
10 & 14 & 18 \\
11 & 04 & 11 \\
12 & 10 & 14 \\
13 & 08 & 16 \\
14 & 08 & 14 \\
15 & 08 & 18 \\
\hline
\end{tabular}

Tabla 2. Puntaje promedio en el pretest y postest

\begin{tabular}{cc}
\hline $\begin{array}{c}\text { Momento de } \\
\text { evaluación }\end{array}$ & $\begin{array}{c}\text { Octavo ciclo de la Escuela Profesional } \\
\text { de Ingeniería Electrónica - UNJFSC }\end{array}$ \\
\hline Pre- test & 10.5 \\
Post - test & 15.9 \\
\hline
\end{tabular}

Figura 7. Histograma y polígono de frecuencia pretest

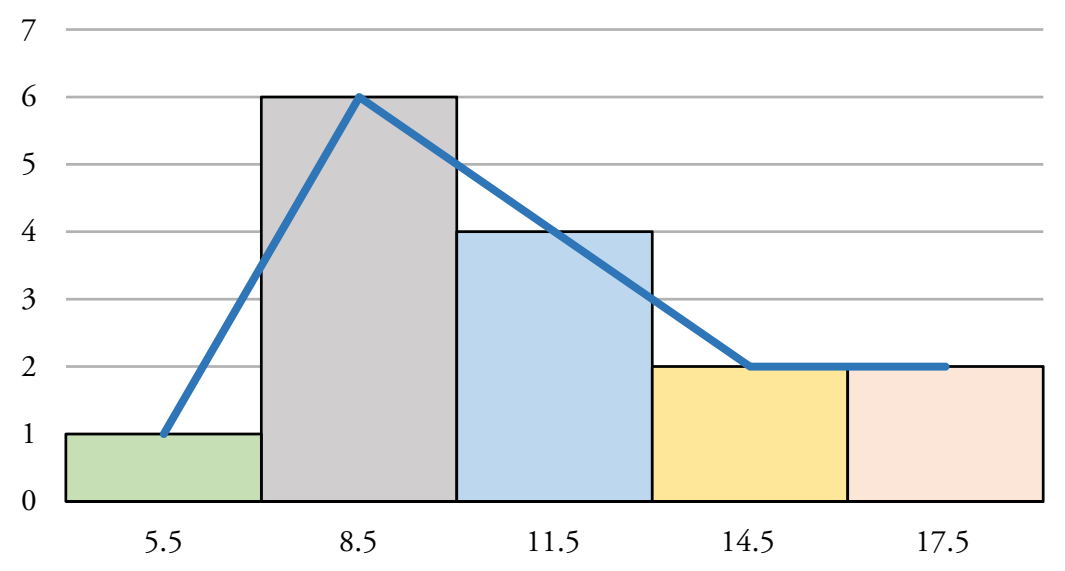


Figura 8. Histograma y polígono de frecuencia postest

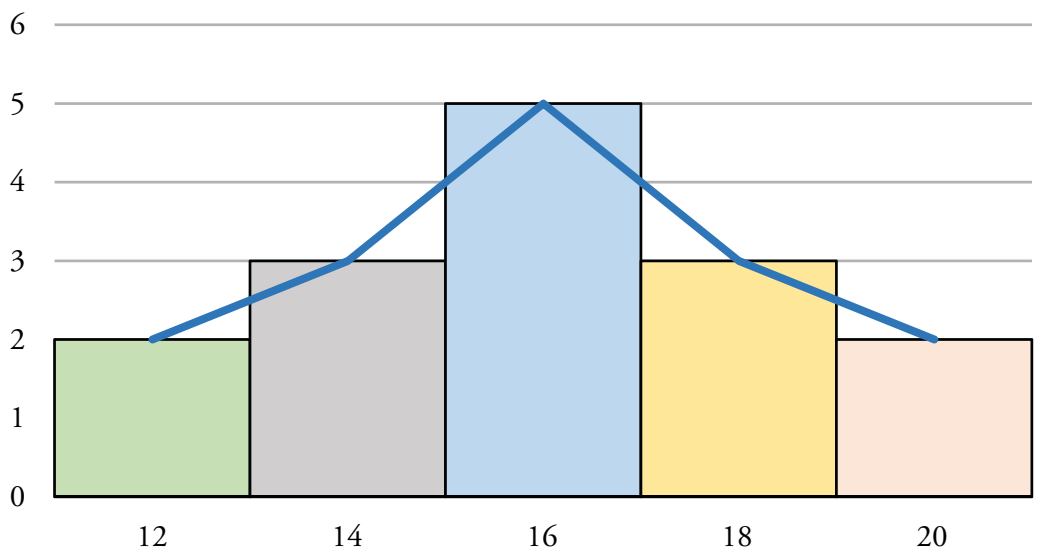

Analizando los histogramas y la Tabla 1, se puede indicar:

Del pretest:

- El 66\% de los estudiantes desaprobó, se obtuvo como nota mínima 04.

- El puntaje más frecuente es de 08 .

Del postest:

- El $100 \%$ de los estudiantes aprobó el test, se obtuvo como nota máxima 20 y mínima 11.

- El puntaje más frecuente es de 16.

Los alumnos realizaron su placa de comparadores (ver Figura 9.a) y placa del microcontrolador (ver Figura 9.b) para el robot seguidor de línea, utilizando el software de diseño Proteus 8.3 logrando buenos resultados. Para el diseño mecánico del robot seguidor de línea (ver Figura 9.c), los estudiantes a su criterio determinaron el mejor modelamiento que favorezca a su robot, para ello, se les indico previamente que investigaran sobre las configuraciones mecánicas para robot móviles. Finalmente, lo estudiantes programaron, realizaron las pruebas de calibración y compitieron con sus robots seguidores de línea (ver Figura 9.d). 
Figura 9. a-Diseño PCB y físico de la placa de sensores. b-Diseño mecánico de los robots seguidor de línea. c- Diseño PCB y físico de la placa del microcontrolador. d-Estudiantes realizando pruebas del robot seguidor de línea

a)

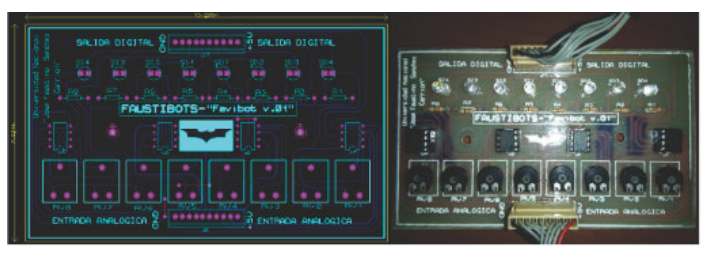

c)

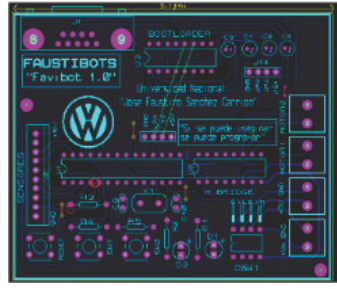

b)

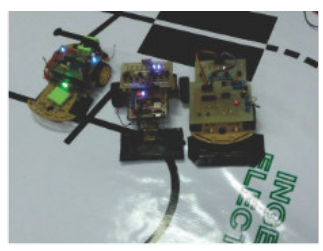

d)

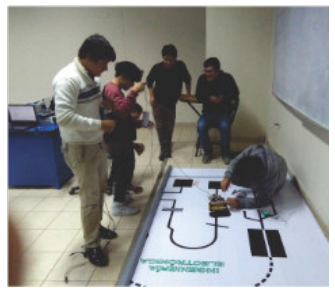

\subsection{Contrastación de hipótesis}

A continuación, se procede a realizar la prueba de hipótesis para determinar y validar lo planteado inicialmente, para ello, se emplea los datos obtenidos en los resultados de la investigación. Se conoce que la muestra es igual a la población total, ya que se está evaluando a cada alumno que pertenece al octavo ciclo (sujetos de estudio), los cuales son 15 en total. Los alumnos que aprobaron el examen en el postest representan el 100\% y nuestra hipótesis a demostrar será la siguiente:

¿Los microcontroladores se relacionan significativamente de forma positiva con la microrrobótica en los estudiantes de octavo ciclo de Ingeniería Electrónica de la Universidad Nacional José Faustino Sánchez Carrión?

Formulando las hipótesis: Hipótesis nula $H_{0}$ y la hipótesis alternativa $H_{1}$

- $H_{0}$ : Los microcontroladores no se relacionan significativamente de forma positiva con la microrrobótica

- $H_{1}$ : Los microcontroladores se relacionan significativamente de forma positiva con la microrrobótica 
Como se trata de una investigación correlacional, se empleará la prueba de correlación de Pearson utilizando los resultados del postest. A continuación, se define dos variables para este trabajo:

- Las notas obtenidas en el postest

- Rango de notas que se emplea en una evaluación

El rango de notas que se emplea en una evaluación están comprendido entre 0 a 20 y se pueden clasificar en los intervalos: deficiente $(0-4)$, bajo $(5-8)$, regular $(9-12)$, bueno $(13-16)$ y excelente $(17-20)$, por lo tanto, se debe establecer una recodificación de variables utilizando el software SPSS, quedando de la siguiente manera deficiente (1), bajo (2), regular (3), bueno (4) y excelente (5). Aplicando la prueba de Pearson a las notas obtenidas del postest y al nuevo rango recodificado de notas entre 0 y 20 se obtiene lo siguiente:

Tabla 3. Correlación notas postest y rango de notas

\begin{tabular}{llcc}
\hline \multicolumn{3}{c}{ Correlaciones } & \\
\hline & & Notas postest & Rango de notas \\
\hline \multirow{3}{*}{ Notas postest } & Correlación de Pearson & 1 &, $931^{* *}$ \\
& Sig. (bilateral) & &, 000 \\
& $\mathrm{~N}$ & 15 & 15 \\
\hline \multirow{5}{*}{ Rango de notas } & Correlación de Pearson &, $931^{* *}$ & 1 \\
& $\mathrm{~N}$ &, 000 & \\
& $\mathrm{~N}$ & 15 & 15 \\
\hline
\end{tabular}

**. La correlación es significativa en el nivel 0,01 (bilateral).

Prueba estadística: coeficiente de correlación de Pearson

Regla de decisión: Si $\mathrm{p} \leq 0.05$ se rechaza $H_{0}$

El valor de "p» en la Tabla 3 es de 0,000, por lo tanto, se rechaza la hipótesis nula $H_{0}$ y se acepta la hipótesis alternativa $H_{1}$. Asimismo, el índice $\mathrm{R}$ de Pearson es de 0,931, esto se interpreta como una muy buena correlación.

Conclusión: Los microcontroladores si se relacionan de forma significativa y positiva con la microrrobótica en el aprendizaje de los estudiantes de octavo ciclo de Ingeniería Electrónica de la Universidad Nacional José Faustino Sánchez Carrión.

\section{DisCUSIÓN Y CONCLUSIONES}

Los resultados encontrados muestran que los estudiantes mejoraron significativamente sus conocimientos con respecto a los microcontroladores esto 
mediante la aplicación de la microrrobótica como una estrategia de enseñanza, coincidiendo así con Romero, et al. (2006), quien afirma que tras varios años de puesta en práctica esa experiencia puede confirmar que los resultados han sido muy satisfactorios.

El proyecto realizado logró incentivar a los estudiantes para que investiguen sobre proyectos similares y obtengan ideas e información complementaria a la que brinda el docente, de esta manera diseńaron e implementaron su robot seguidor de línea.

Finalmente, se señala que los microcontroladores guardan una relación significativa y positiva con la microrrobótica, ya que conforman una parte fundamental del mecanismo robótico, siendo así el que controla sus movimientos y realiza funciones o tareas determinadas sin necesidad de la intervención de un operario, lo cual le permite que sea considerado autónomo basándose solo en la programación que se le ingrese. Por otro lado, esto asegura también una relación como método de aprendizaje para el estudiante, tal como lo afirma Díaz et al. (2013) quien, mediante el uso de sensores, emisores de luz y de sonidos, basados en la programación de Microcontroladores, diseñó estrategias didácticas y confeccionó guías de trabajo para las y los estudiantes.

\section{REFERENCIAS BIBLIOGRÁFICAS}

Angulo, I. y Angulo, J. (2003). Microcontroladores PIC Diseño práctico de aplicaciones Primera parte. Madrid: McGraw-Hill Interamericana.

Arias, M., Rodríguez, A., Lamar, D., Linera, F. y Hernando, M. (2010). Influence of PBL Practical Classes on Microcontroller-Based Digital Systems Learning. IEEE EDUCON 2010 Conference, Madrid, 1777-1782. https:// doi.org/10.1109/EDUCON.2010.5492418

Bolton, W. (2006). Mecatrónica Sistemas de control electrónico en Ingeniería Mecánica y Eléctrica. Recuperado de http://www.freelibros.org/libros/ mecatronica-w-bolton-alfaomega2\%C2\%B0-edicion.html

Bono, A. y Martín, B. (2003). Enseñanza de una metodología para la programación de microcontroladores en el marco de titulación de Electrónica Industrial. Recuperado de http://www.epsevg.upc.edu/xic/ponencias/R0189.pdf

Cifuentes, A. (2011). Didáctica para la enseñanza de microcontroladores a estudiantes de ingeniería mecatrónica (Tesis de Maestría). Universidad Militar de Nueva Granada, Bogotá, Colombia.

Contreras, L. (2014). Diseño y construcción de robot seguidor de línea (Tesis de pregrado). Universidad Tecnológica de Querétano, Santiago de Querétaro, México. 
Cruz, A. (2017). El aprendizaje basado en proyectos como una estrategia docente para el desarrollo de competencias profesionales en estudiantes de Ingeniería Mecánica (Tesis de Maestría). Instituto Politécnico Nacional Secretaría de Investigación y Posgrado, Ciudad de México, México.

Díaz, A., Jeraldo, R. y Tapia, A. (2013). Montaje de sensores y programación de Microcontroladores con una propuesta didáctica fundada en la enseñanza de las ciencias basadas en la indagación (ECBI) para el estudio de la fisica en educación media (Tesis de pregrado). Universidad de Santiago de Chile, Santiago, Chile.

Galeana, L. (2006). Aprendizaje basado en proyectos. Recuperado de unisurabp. blogspot.com/2010/11/concepto-de-abp.html

Macías-Guarasa, J., Montero, J., San-Segundo, R., Araujo, A. y Nieto-Taladriz, O. (2006). A project-based learning approach to design electronic systems curricula. IEEE Transactions on Education, 49(3), 389-397. https://doi. org/10.1109/TE.2006.879784

Microchip (s.f.). Microcontroller PIC16F876A. Recuperado de: https://www. microchip.com/wwwproducts/en/PIC16F876A

Microsystems Engineering (2005). Microbot Home Boe-Bot. Recuperado de http:// documents.mx/documents/manual-del-home-boe-bot-en-castellano.html

Morales, P. y Landa, V. (2004). Aprendizaje basado en problemas problema-based learning. Theoria, (13), 145-157.

Najid, N., Ridzwan, R. y Tee, T. (2018). A Review of Problem-Based Learning in Electronic Engineering Course. International Journal of Academic Research in Business and Social Sciences, 7(12), 39-47. https://doi.org/10.6007/ IJARBSS/v7-i12/3590

Olivares, G. (2011). Diseño, construcción y control de un MicroRobot SACARA Industrial de Alta Precisión. México: Instituto Politécnico Nacional.

Oliver, J., Toledo, R., Pujol, J., Sorribes, J. y Valderrama, E. (2009). Un ABP basado en la robótica para las ingenierías. Recuperado de http://hdl.handle. net $/ 2099 / 7878$

Reyes, C. (2008). Microcontroladores PIC Programación en Basic. Recuperado de https://es.slideshare.net/KevinAvilesTorres1/microcontroladores-picbasic-carlos-a-reyes-70827706

Romero, S., Angulo, I. y Angulo, J. (2006). La microrobótica como estrategia de enseñanza-aprendizaje y evaluación del uso de microcontroladores. Recuperado de taee.euitt.upm.es/actas/2006/papers/2006S1D04.pdf

Sababha, B., Alqudah, Y., Abualbasal, A. y AlQaralleh, E. (2016). Project-Based Learning to Enhance Teaching Embedded Systems. Eurasia Journal of Mathematics, Science and Technology Education, 12(9), 2575-2585. https:// doi.org/10.12973/eurasia.2016.1267a 